\title{
Mechanical evaluation of cerebral aneurysm clip scissoring phenomenon: comparison of titanium alloy and cobalt alloy
}

\author{
Keiji Tsutsumi, Tetsuyoshi Horiuchi, Kazuhiro Hongo \\ Department of Neurosurgery, \\ Shinshu University School of Medicine, Matsumoto, Japan
}

Correspondence to:

Tetsuyoshi Horiuchi, M.D.

Department of Neurosurgery,

Shinshu University School of Medicine,

3-1-1 Asahi, Matsumoto, 390-8621, Japan

Telephone: $+81-263-37-2690$

Fax: $+81-263-37-0480$

E-mail: tetuyosi@shinshu-u.ac.jp

ORCID of the correspondence author is 0000-0001-5620-510X 


\begin{abstract}
Cerebral aneurysm clip blades crossing during surgery is well known as scissoring. Scissoring might cause rupture of the aneurysm due to laceration of its neck. Although aneurysm clip scissoring is well known, there have been few reports describing the details of this phenomenon. Quasi-scissoring phenomenon was introduced mechanically by rotating the clip head attached to a silicone sheet. The anti-scissoring torque during the twist of the blades was measured by changing the depth and the opening width. The closing force was also evaluated. Sugita straight clips of titanium alloy and cobalt alloy were used in the present study. In both materials, the anti-scissoring torque and the closing force were bigger $3 \mathrm{~mm}$ in thickness than $1 \mathrm{~mm}$. The initial closing forces and the anti-scissoring torque values at each rotation angles were increased in proportion to depth. Closing forces of titanium alloy clip were slightly higher than those of cobalt alloy clip. By contrast, anti-scissoring torque values of cobalt alloy clip were bigger than those of titanium alloy clip in all conditions. In condition of $3 \mathrm{~mm}$ in thickness and $3 \mathrm{~mm}$ in depth, anti-scissoring torque vales of titanium alloy clip decreased suddenly when an angle surpassed 70 degrees. Aneurysm clip scissoring phenomenon tends to occur when clipping the aneurysm neck only with blade tips. Based on the results of this experiment, titanium alloy clip is more prone to scissoring than cobalt alloy clip under the condition that the wide blade separation distance and the shallow blade length.
\end{abstract}

Key words: scissoring phenomenon, cerebral aneurysm clip, clipping, titanium alloy, cobalt alloy 


\section{Introduction}

Although scissoring phenomenon is a well-known malfunction of cerebral aneurysm clip, detailed factors of this phenomenon has not been well understood. If the aneurysm clip scissoring occurs during a clipping procedure, not only the closure of the aneurysm neck becomes insufficient, but also there is a possibility of causing rupture of the aneurysm. Although pure titanium and titanium alloy have many excellent characteristics including high specific strength, high corrosion resistance, high biocompatibility, and non-magnetic non-toxic property [1], there have been several reports of scissoring with titanium and titanium alloy cerebral aneurysm clips [2-5]. Therefore, it is essential for neurosurgeons to correctly understand the characteristics of titanium and titanium alloy as well as knowing the functional properties of the aneurysm clips. In this study, we measured anti-scissoring torques and closing forces mechanically and compared cobalt with titanium alloy clip.

\section{Materials and Methods}

Measurement of closing force: Measurement of closing forces of aneurysm clip was used a clip closing force meter $[6,7]$. As specific measurement conditions, the closing forces from the blade tips were measured in $3.0 \mathrm{~mm}$ increments $(3,6$, and $9 \mathrm{~mm})$ at the two blade separation distances (1 and $3 \mathrm{~mm})$ (Fig. 1).

Measurement of anti-scissoring torque: Quasi-scissoring phenomenon was introduced mechanically by rotating the clip head using our own manufactured torque measurement 
system [8]. Torque measurement method was reported previously [8]. Our previous study [8] showed that torque values increased in parallel with clip head rotation and decreased at around 90 degrees when clip blades were crossed. We defined as anti-scissoring strength just before blade twisting (Fig. 2). The anti-scissoring torques with changing the depth (3, 6 , and $9 \mathrm{~mm}$ ) and the opening width (1 and $3 \mathrm{~mm})$ were measured.

Aneurysm clips: Sugita titanium alloy (Titanium II ) and cobalt alloy (Elgiloy) straight clips of $18 \mathrm{~mm}$ in blade length (Mizuho Co., Ltd., Tokyo, Japan) were used and compared. All clips were offered in a brand-new state by a manufacturer. The blade lengths and structure of both clips are same, but the shape of the clip head and the blades are a little different (Fig. 3).

Statistical analysis: Analysis was performed by Statistical Package for Social Sciences (SPSS) version 23 software. All data are presented as the means \pm standard deviation and $n$ represents the number of the clips. Differences are considered significant at a $\mathrm{P}$ value less than 0.05 . Unpaired t test or ANOVA with post hoc Tukey's test were used as appropriate.

\section{Results}

Closing force: The closing force near the tips of the blades was the lowest and it showed higher values closer to the coil spring (Fig. 4). Closing forces of both materials (titanium alloy and cobalt alloy) were higher $3 \mathrm{~mm}$ of opening width than $1 \mathrm{~mm}(P<0.05)$ (Fig. 4). 
Aneurysm clip scissoring

Under each condition of the opening width, the increase in the closing force of the straight clips of both materials was linear (Fig. 4).

Anti-scissoring torque: The torque values of both materials increased with the rotation angle. Moreover, anti-scissoring torque of both materials was higher as the blade length from the blade tips was longer $(P<0.05)$ (Fig. 5). Additionally, the torque values of both materials were greater $3 \mathrm{~mm}$ in thickness than $1 \mathrm{~mm}(\mathrm{P}<0.05)$ (Fig. 6). Under either condition, although anti-scissoring torque of titanium alloy clip was less than that of cobalt alloy clip, basically the torque value rises linearly up to around 90 degrees (Fig. 5, 6, 7). However, under the condition that the blade separation distance was wide (opening width in $3 \mathrm{~mm}$ ) and the blade length from the blade tips was shallow (depth in $3 \mathrm{~mm}$ ), anti-scissoring torque value of the titanium alloy clip sharply decreased near 70 degrees (Fig. 5a, 6a, 7).

Clip material: Titanium alloy clips were slightly higher in the closing force than cobalt alloy clips (Fig. 4). By contrast, torque values of cobalt alloy clip were more than those of titanium alloy clip $(\mathrm{P}<0.05)$ (Fig. 7). Notably, under the conditions of $3 \mathrm{~mm}$ in thickness with $3 \mathrm{~mm}$ in depth, torque values of titanium alloy clip decreased suddenly when rotation angles exceeded 70 degrees (Fig. 5a, 6a, 7).

\section{Discussion}

Even though endovascular treatment has emerged as a treatment for cerebral 
aneurysms, all aneurysm cannot be obliterated with endovascular approach. Microsurgical neck clipping is still useful treatment. Various elements are related to accomplish sufficient aneurysm neck clipping. Thorough understanding of material differences and functional properties of the cerebral aneurysm clips are major elements for safe and secure use of aneurysm clip. As an element of aneurysm side, the thickness of the aneurysmal wall, the presence or absence of arteriosclerosis of the aneurysm neck, and the size of the aneurysm are important. We have reported some experiments in order to understand the functional properties of cerebral aneurysm clip such as $[3,6-8]$ the difference in the closing force by the position and form of the blade [6], the torque value at the blade position to prevent scissoring [8], and the blade opening width depending on the type of clip [7]. In this study, to evaluate the influence of aneurysm side such as the thick wall, the presence of arteriosclerosis, and large size, we measured the closing force and the anti-scissoring torque by varying the opening width of clip blades. Moreover, using two materials of Sugita clip, we examined and compared the difference in the material.

\section{Metallic difference}

Sugita titanium clips are made of titanium alloy containing $6 \%$ aluminum and $4 \%$ vanadium (Ti-6Al-4V). Ti-6Al-4V is light and high specific strength but weak against bending and easily cracked. According to the American Society for Testing Materials (ASTM) standards [9] which observes the resistance to the drawing force of the metal wire, Ti-6Al-4V has only about half the stickiness of the cobalt alloy (Elgiloy) and represents the low stickiness of the titanium alloy. Generally, a metal with high stiffness and high strength 
seems to be difficulty deformed. The stiffness of the metal can be evaluated by Young's modulus, while the strength of the metal can be measured by tensile strength and yield strength. The metal stiffness (Young's modulus), tensile strength and yield strength of titanium alloy and cobalt alloy are $100 \mathrm{GPa}$ and $181 \mathrm{GPa}, 860 \mathrm{MPa}$ and $1450 \mathrm{MPa}, 795 \mathrm{MPa}$ and $1300 \mathrm{MPa}$, respectively.

\section{Closing force}

A coil spring part determines the closing force. Titanium alloy is inferior to cobalt alloy in terms of metal stiffness and strength. Therefore, it was assumed that the closing force of titanium alloy clip was lower than that of cobalt alloy clip. However, in the present experiment, the closing force of titanium alloy clip was slightly higher than cobalt alloy clip, irrespective of opening width (Fig. 4). Horiuchi et al. [7] reported similar results in a long clip of $25 \mathrm{~mm}$ in blade length. Although Sugita clips of both materials have basically the same structure, slight differences are seen in the shape of the coil spring and the shape of the blades (Fig. 3), which might be related to the result of the closing force.

\section{Anti-scissoring torque}

A crossover part has a function for preventing scissoring [8]. Sugita aneurysm clip adopts a stabilizing bridge wire mechanism as a scissoring prevention system [8]. By contrast, Yasargil clip adapts a box lock mechanism. Regarding the anti-scissoring torque, the cobalt alloy clip was consistently superior to the titanium clip under all conditions (Fig. 5). Metal stickiness, stiffness, and strength are thought to be a major cause of the difference 
in anti-scissoring torque. The anti-scissoring torque of the cobalt alloy clip linearly increased by clip head rotation at 3,6,9 $\mathrm{mm}$ in depth with 1 and $3 \mathrm{~mm}$ in opening width (Fig. 5b). However, under the condition that the blade separation distance was wide (opening width in $3 \mathrm{~mm}$ ) and the blade length from the blade tips was shallow (depth in 3 $\mathrm{mm}$ ), torque values of the titanium alloy clip sharply decreased at the top of the vicinity of 70 degrees (Fig. 5a, 6a, 7). With clipping of aneurysm without thick wall, severe arteriosclerosis, or large size, the titanium alloy clip seems to be functionally equivalent to cobalt alloy clip. However, based on this experiment, titanium alloy clip was more likely to cause scissoring in wide opening of clip blades and shallow clipping (Fig. 5a, 6a, 7).

\section{Conclusions}

Under various conditions, we have examined and compared closing forces and antiscissoring torques of Sugita clips (made of titanium alloy and cobalt alloy). Based on our study, aneurysm clip scissoring phenomenon tends to occur with blade-tip clipping. Especially, in atherosclerotic aneurysm and/or large aneurysm, more deeper clipping is necessary when using titanium alloy clip. Additionally, parallel clipping technique, booster clipping or cobalt alloy clipping should be considered for atherosclerotic and/or large aneurysm. 
Aneurysm clip scissoring

\section{Disclaimer Statements}

Funding There are no funding for this article.

Conflicts of interest The authors declare that they have no conflict of interest. 


\section{References}

1 Lawton MT, Ho JC, Bichard WD, Coons SW, Zabramski JM, Spetzler RF. Titanium aneurysm clips: Part $1-$ mechanical, radiological, and biocompatibility testing. Neurosurgery. 1996;38:1158-64.

2 Hirashima Y, Kurimoto M, Kubo M, Endo S. Blade crossing of a pure titanium clip applied to a cerebral aneurysm - case report. Neurol Med Chir (Tokyo). $2002 ; 42: 123-4$

3 Horiuchi T, Li Y, Seguchi T, Sato A, Aoyama T, Hanaoka Y, Hongo K. Clip blade scissoring with titanium bayonet clip in aneurysm surgery. Two case reports. Neurol Med Chir (Tokyo). 2012;52:84-6.

4 Nievas MN, Hollerhage HG. Risk of intraoperative aneurysm clip slippage: a new experience with titanium clips. J Neurosurg. 2000;92:478-80.

5 Papadopoulos MC, Apok V, Mitchell FT, Turner DP, Gooding A, Norris J. Endurance of aneurysm clips: mechanical endurance of Yasargil and Spetzler titanium aneurysm clips. Neurosurgery. 2004;54:966-70.

6 Horiuchi T, Rahmah NN, Yanagawa T, Hongo K. Revisit of aneurysm clip closing forces: comparison of titanium versus cobalt alloy clip. Neurosurg Rev. $2013 ; 36: 133-8$

7 Horiuchi T, Ito K, Hongo K, Shibuya M. Mechanical evaluation of long titanium alloy 
clip - comparison of cobalt alloy clip. Neurol Med Chir (Tokyo). 2014;54: 176-9.

8 Horiuchi T, Hongo K, Shibuya M. Scissoring of cerebral aneurysm clips: mechanical endurance of clip twisting. Neurosurg Rev. 2012;35:219-25.

9 American Society for Testing and Materials (ASTM) Designation. Annual Book of ASTM Standards, Section 13, Medical Devices and Services, Volume 13.01 Medical and Surgical Materials and Devices (I): E667 - F2477. West Conshohocken, PA: ASTM Inter national, 2016

\section{Figure legends}

Figure 1: Measurement of closing forces and anti-scissoring torques were made with changing the blade separation distance $(1$ and $3 \mathrm{~mm})$ and blade length from the blade tips (3, 6 , and $9 \mathrm{~mm}$ ) respectively.

Figure 2: Effects of clip rotation angle on torque value during Sugita titanium straight clip head rotation at a depth of $3 \mathrm{~mm}$ and a thickness of $1 \mathrm{~mm}(n=5)$. Titanium II straight clips (17-001-18).

Figure. 3: Photographs of Sugita cobalt alloy (07-940-18) (A and B) and titanium alloy clips (17-001-18) (C and D).

Figure. 4: Effects of distance from blade tips on Titanium II (17-001-18) and Elgiloy 
(07-940-18) straight clips closing forces $(n=5)$ : Square indicates the blade separation distance is $1 \mathrm{~mm}$. Circle indicates the blade separation distance is $3 \mathrm{~mm}$. Asterisk and dagger indicate significant differences between blade separation distance $1 \mathrm{~mm}$ and $3 \mathrm{~mm}$ for Elgiloy and Titanium II , respectively $(P<0.05)$.

Figure. 5: Effects of distance from blade tips on torque value during clip head rotation at a thickness of $3 \mathrm{~mm}(n=5)$. a Titanium II straight clips (17-001-18) and b Elgiloy (07-940-18). Asterisk and dagger indicate significantly different from depths of 3 and $6 \mathrm{~mm}$, respectively $(P<0.05)$.

Figure. 6: Effects of blade separation distance on torque value during clip head rotation at a depth of $3 \mathrm{~mm}(n=5)$. a Titanium II straight clips (17-001-18) and b Elgiloy (07-940-18). Asterisk indicates significant differences between thickness of 1 and $3 \mathrm{~mm}(P<0.05)$.

Figure. 7: Effects of distance from blade tips on torque value during clip head rotation at a depth of $3 \mathrm{~mm}$ and thickness of $3 \mathrm{~mm}(n=5)$. Titanium II versus Elgiloy Sugita straight clips $(n=5)$.

Asterisk indicates significant differences between Titanium II and Elgiloy $(P<0.05)$. 
Figure. 1

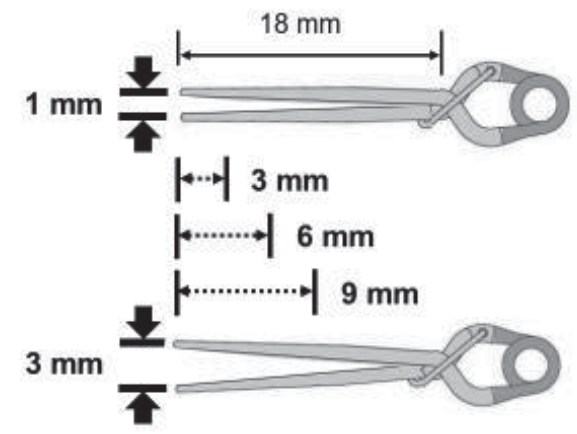

Figure. 2

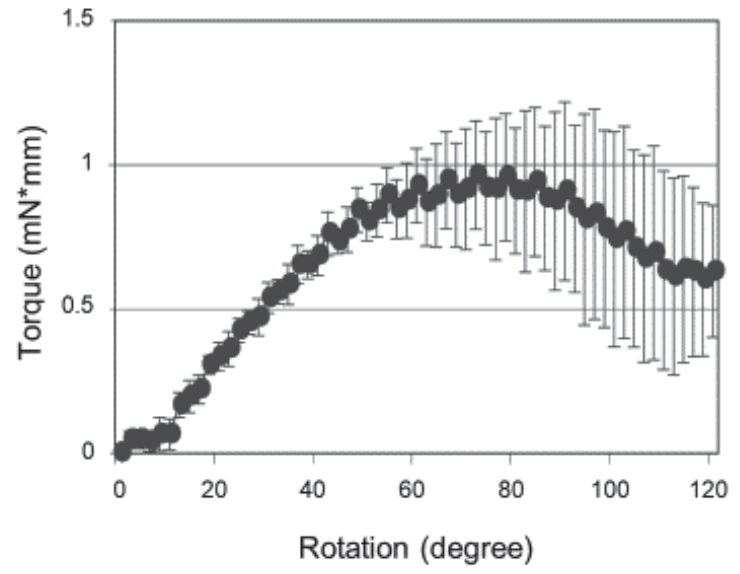


Figure. 3

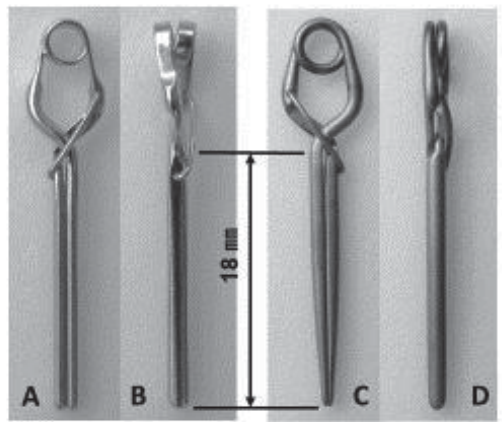

Figure. 4

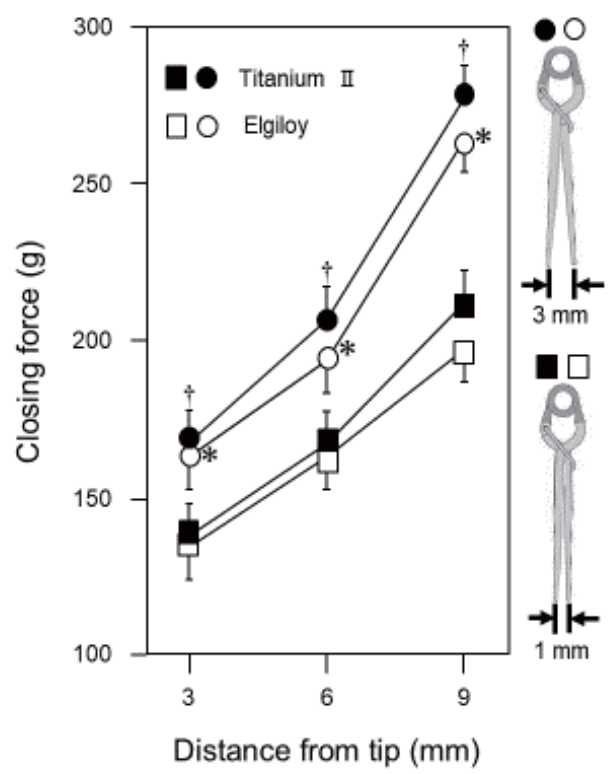


Figure. 5a

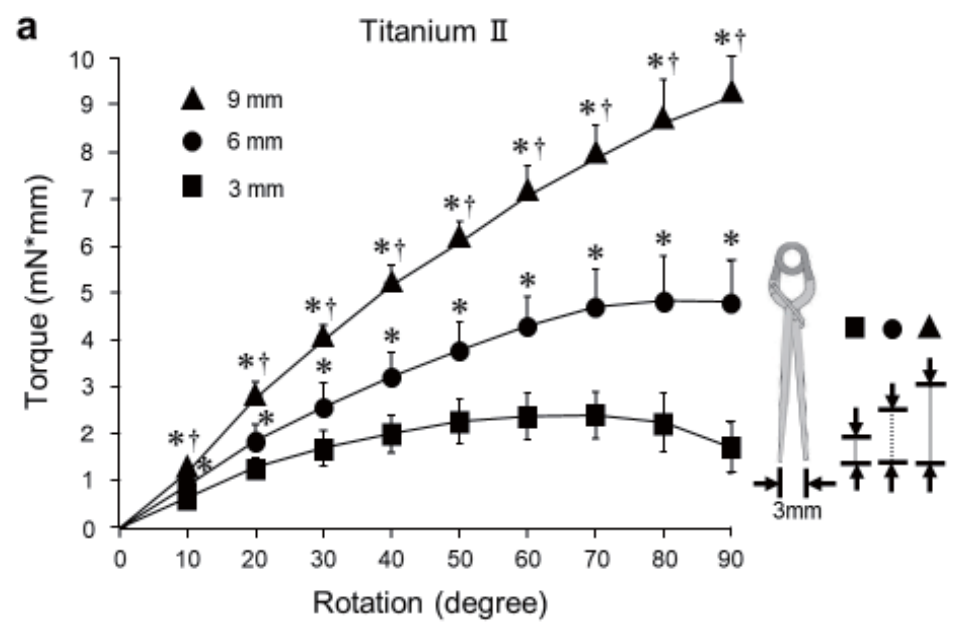

Figure. 5b

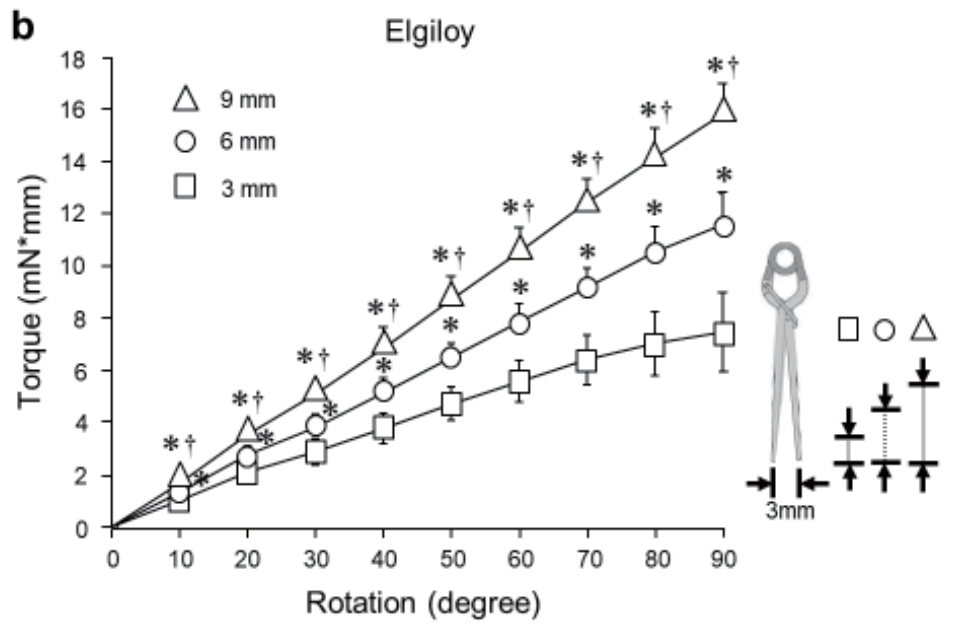


Figure. 6a

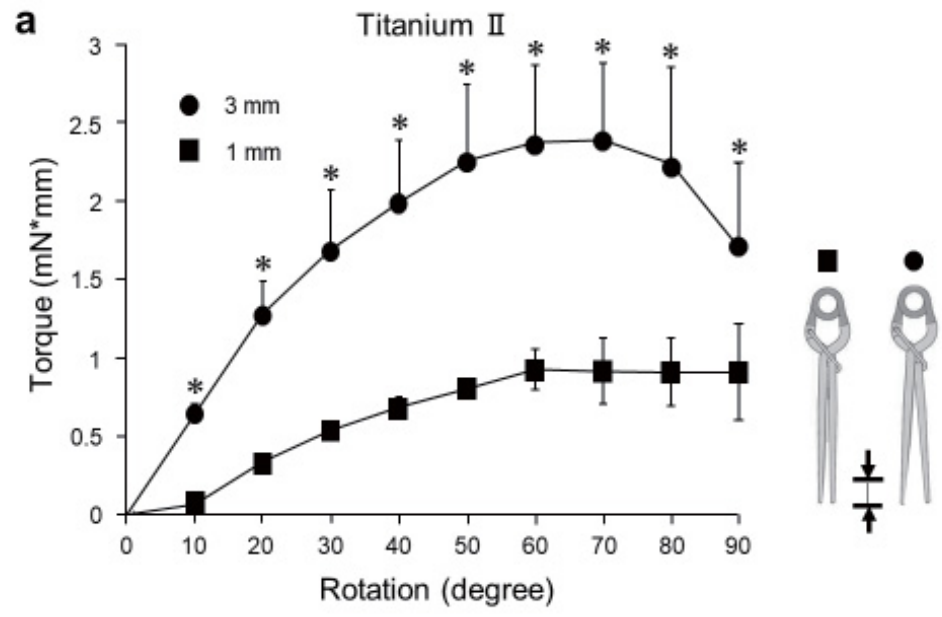

Figure. $6 b$

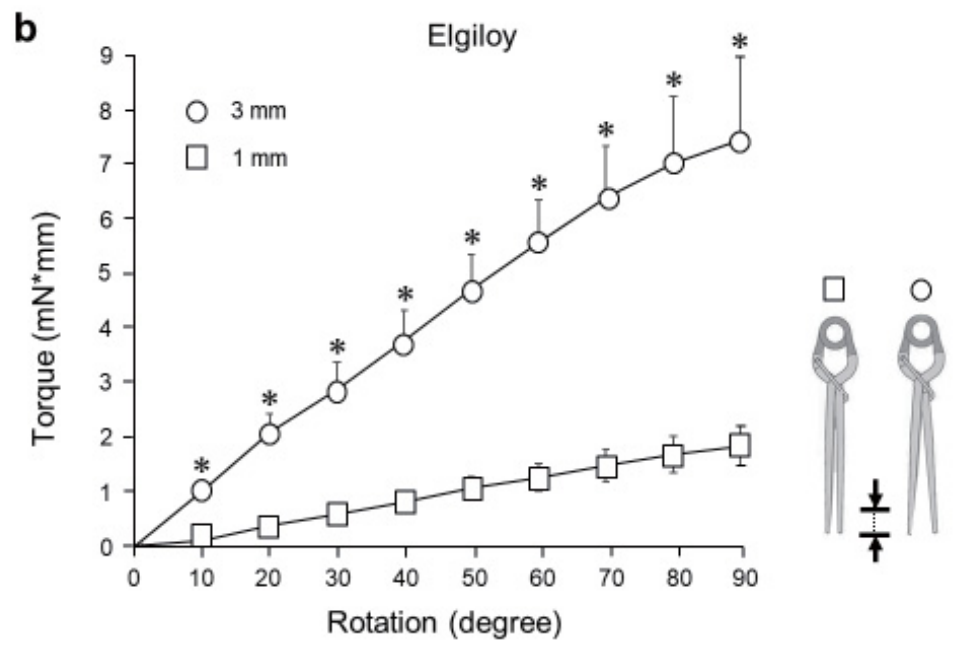


Figure. 7

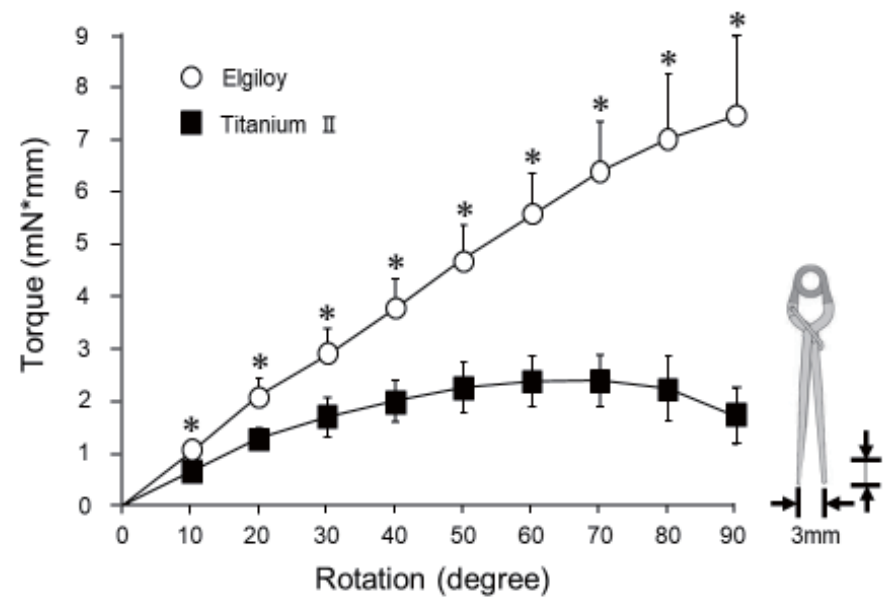

